\title{
Ujinsi wa nomino za mkopo katika lugha ya Kiswahili
}

\author{
Arnold, B. G. Msigwa ${ }^{1}$ \\ University of Daar es Salaam
}

\begin{abstract}
Ikisiri
Kwa kuzingatia taipolojia ya lugha duniani kijinsi, lugha zimegawika katika makundi makuu matatu: lugha zenye jinsi 'ke', lugha zenye jinsi 'me' na lugha zisojinsi, yaani zile ambazo hazina jinsi. Lugha nyingi za Kibantu, Kiswahili kikiwa miongoni mwazo, huchukuliwa kuwa ni lugha zisojinsi kwa maana kwamba hazina ujinsi. Hata hivyo, hitimisho hili linaelekea kuwa tenge kwa namna fulani, hususani tukizingatia nomino za mkopo zilizoswahilishwa. Makala haya yatatumia data za kiuchunguzi kutoka kwenye baadhi ya nomino za mkopo ili kulithibitisha dai hili na kuujengua mtazamo unaokichukulia Kiswahili kuwa ni lugha isojinsi. Swali linaloulizwa na makala haya ni je, nomino za mkopo zinazotumika tofauti kutegemea jinsi husika yaani jinsi 'me' au jinsi 'ke' zinapoingizwa katika lugha ya Kiswahili huchukua tabia gani? Je, kwa kuzingatia tabia ya nomino hizo, bado ni sahihi kuhitimisha kwa ujumla kuwa lugha ya Kiswahili ni lugha isojinsi? Maswali haya na mengine yenye uelekeo kama huu ndiyo yatashughulikiwa katika makala haya. Data zitakusanywa kutoka uwandani kwa njia ya mahojiano na ushuhudiaji.
\end{abstract}

Dhana muhimu: Jinsi, isojinsi.

\begin{abstract}
Masculine, the world languages are generally categorized into two categories; masculine language, feminine language and neuter language. Many Bantu Languages, Swahili inclusive is generally categorized as neuter language; meaning that objects have on its vocabulary like noun has no grammatical gender. However, if you underscore a close observation especially on the borrowed noun, you find something different. The aim of the current article therefore is to investigate how the borrowed noun that enters into Swahili from gendered language behaves. The questions raised by this article are: first, do really borrowed noun that come from gendered language into Swahili become neuter like other noun? Secondly, by looking those borrowed now from gendered language and the way they behaves in Swahili, is it correct to generalize that Swahili language is neuter. These questions and other of this trend are the ones addressed in this article. Data collected from through interview and observation is used to support the argument.
\end{abstract}

Keywords: masculine, neuter

\section{Utangulizi}

Kwa ujumla neno jinsi katika lugha hutofautisha kati ya a-kiume na a-kike, kwa mfano binti yangu na kijana wangu ni wakulima. Kulingana matumizi ya maneno haya katika Utamaduni wa waswahili, kijana ina rejelea jinsi ya kiume na binti inarejelea jinsi ya kike. Makala haya yanasaili

\footnotetext{
${ }^{1}$ Arnold B. G. Msigwa is a Kiswahili lecturer at the University of Dar es Salaam in the Department of Kiswahili Language and Linguistics. He holds an MA and PHD degrees from the University of Dar es Salaam respectively. He has specialised in Second Language Learning and authored several articles. His research interest includes applied linguistics, lexicography and morphology. E-mail: agawasike@gmail.com
} 
mtazamo wa ujumla unaochuchukulia kuwa lugha ya Kiswahili ni lugha isojinsi ${ }^{2}$. Nia hasa ya usaili huu ni kuchochea fikra miongoni mwa wanaisimu kuhusu taipolojia ya lugha za Kibantu. Msukumo wa kuandika makala haya umetokana na halisi halisi inayoonekana katika jamii ya Waswahili ambapo watu wanapewa majina yanayoelekea kutokuwa na asili za lugha za Kiafrika. Baadhi ya majina hayo yakichunguzwa etimolojia yake yana asili moja lakini yanavyotumika katika lugha ya Kiswahili yanaashiria jinsi mbili tofauti. Mifano kadhaa itatumika ili kushadidia hoja yetu.

\section{Usuli wa Kiisimu wa Lugha ya Kiswahili}

Wataalamu mbalimbali wa masuala ya historia ya lugha kama vile; Amidu (1995) na Massamba (2002: 2007) wanaonesha kwamba Kiswahili ni lugha ya Kibantu yenye chimbuko lake katika Pwani ya Afrika Mashariki. Kutokana na kukaribiana kwake kwa muda mrefu na lugha zingine kama vile Kiarabu, Kiingereza na kadhalika, Kiswahili kimechukua baadhi ya maneno kutoka lugha hizo na kuyaingiza katika msamiati wake. Aidha, kumekuwa na mwingiliano wa dini ya Kikristo na Kiislamu ambao pia unaelekea kuleta athari za kimsamiati katika lugha ya Kiswahili. Athari za kimsamiati zinazoletwa na mwingiliano huu wa kidini zinaelekea kuifanya lugha ya Kiswahili ianze kuwa na tabia ya lugha zenye jinsi ndani ya baadhi ya msamiati wake wa mkopo. Msamiati wa mkopo ni aina ya maneno yaliyochukuliwa kutoka kwenye lugha nyingine (lugha kopwaji) na kuingizwa na kutumiwa katika lugha nyingi (lugha kopaji). Mchakato wenyewe wa kuchukua msamiatia kutoka kwenye lugha kopwaji na kuingiza katika lugha kopaji hujulikana mchakato wa ukopaji. Ukopaji huu unaweza kuleta athari za namna fulani za kiisimu kwenye lugha kopaji, kama vile athari za kimaana, kimuundo na kiumbo.

\section{Kategoria ya Lugha za Kijinsi}

Uchunguzi wa masuala ya jinsi na lugha na athari zake katika mahusiano ya kijamii ulianza muda mrefu, hata hivyo kama taaluma iliimarika zaidi miaka ya 1960 na 1970 . Kwa mujibu wa Unger na Crawford (1992), dhana ya ujinsi katika isimu ilianza tangu kwa wanafilosofia wa mwanzo yaani Wagiriki. Kwa mfano mwanafalsafa Protogoras aligawa msamiati wa lugha katika kategoria tatu, yaani jinsi 'ke', jinsi 'me' na isojinsi (-). Lugha nyingi duniani zinaashiria ujinsi katika misamiati yake. Kulingana na Hultgren, (2017) kwa uasili wake, dhana ya jinsi katika lugha ililenga kuangalia namna vipengele vya kisarufi vilivyokuwa vimeambatana katika msamiati fulani wa lugha vilivyowatofautisha watu kwa kuzingatia jisni zao. Hata hivyo, kwa sasa mtazamo umepanuka zaidi kwa jumuisha masuala mengine ya kijamii, kiuchumi, kiutawala na kiumiliki. Kwa mfano kijamii, jinsi inahusishwa na kuzaa na kulea watoto kuwa ni jukumu sawa kati ya mke na mume. Kadhalika kiuchumi, suala la jinsi linahusishwa na uzalishaji na umiliki wa mali kati ya jinsi mbili kwa kutaja kwa uchache. Makala haya hayaingii katika mgogoro huo, bali yanaonesha namna ambavyo lugha ya Kiswahili inaanza kuchukua tabia ya ujinsi kwa kuangalia maneno ya mkopo kutoka lugha zingine. Kwa ujumla, lugha zimegawanyika kijinsi katika makundi matatu: lugha zenye jinsi 'me', lugha zenye jinsi 'ke' lugha zisojinsi. Mgawanyo huu hauwahusu watu pekee yao bali unajumuisha pia viumbe wengine kama vile wanyama na ndege. Jedwali lifuatalo linaonesha lugha zisokuwa na jinsi.

Jedwali Na. 1.1:

Lugha zisojinsi

\begin{tabular}{|c|l|l|l|}
\hline Na. & Lugha & Jinsi & Mahali ilipo \\
\hline 1. & Kiswahili & $(-)$ & Afrika \\
\hline 2. & Kiyoruba & $(-)$ & Afrika \\
\hline 3. & Kihangari & $(-)$ & Ulaya \\
\hline 4. & Kifini & $(-)$ & Asia \\
\hline 5. & Kibengali & $(-)$ & Asia \\
\hline
\end{tabular}

${ }^{2}$ Isojinsi ni tafsiri ya kisawe cha Kiingereza Non-masculine kwa maana ya lugha isiojinsi. 


\begin{tabular}{|c|l|l|l|}
\hline 6. & Kiarmenia & $(-)$ & Ulaya \\
\hline 7. & Kijapani & $(-)$ & Asia \\
\hline 8. & Kichina & $(-)$ & Asia \\
\hline 9. & Kiajemi & $(-)$ & Asia \\
\hline 10. & Kituruki & $(-)$ & Ulaya \\
\hline
\end{tabular}

Chanzo: Utafiti (2018)

Kama jedwali Na. 1.1 hapo juu linavyoonesha kuwa lugha ya Kiswahili ipo kwenye kundi la lugha zisojinsi. Hii ina maana kwamba, katika nomino za lugha ya Kiswahili, hakuna ujinsi ambao unaweza kutombulisha jinsi 'me' au 'ke'. Swali ni je, nomino za mkopo zilizoingia katika lugha ya Kiswahili haiku ikoje? Jedwali Na. 1.2 linaonesha mfano wa lugha ambazo zina ujinsi asilia katika msamiati wake.

Jedwali Na. 1.2

Lugha zenye jinsi katika Msamiati

\begin{tabular}{|c|l|l|l|}
\hline Na. & Jina la lugha & Jinsi & Mahali ilipo \\
\hline 1. & Kiarabu & $(+)$ & Asia \\
\hline 2. & Kihindi & $(+)$ & Asia \\
\hline 3. & Kiamhariki & $(+)$ & Afrika \\
\hline 4. & Kifaransa & $(+)$ & Ulaya \\
\hline 5. & Kiitaliano & $(+)$ & Ulaya \\
\hline 6. & Kireno & $(+)$ & Ulaya \\
\hline 7. & Kihispaniola & $(+)$ & Ulaya \\
\hline 8. & Kirusi & $(+)$ & Ulaya \\
\hline 9. & Kijerumani & $(+)$ & Ulaya \\
\hline 10. & Kiebrania & $(+)$ & Asia \\
\hline
\end{tabular}

Chanzo: Utafiti (2018)

Jedwali $\mathrm{Na}$. 1.2 linaonesha kuwa lugha nyingi za ulaya ndizo zenye ujinsi. Hali inaelekea kuonesha kuwa lugha nyingine ambazo hazina jinsi katika uasili wake, lakini zimekuwa katika makutano na lugha za ulaya, zinaweza kuchukua tabia za msamiati wenye ujinsi. Sehemu ifuatayo inaonesha mifano ya nomino za mikopo zenye ujinsi katika lugha ya Kiswahili.

\section{Ukopaji wa Msamiati katika Lugha}

Kwa mujibu wa Tsvetkov na Dyer (2016, uk 63), ukopaji ni mchakato wa kuhamisha vipengele vya kiisimu vya lugha moja kwenda kwenye lugha nyingine ambao unaweza kuhusisha vipengele vya kimsamiati kifonolojia, kimofolojia na kisintaksia. Tsvetkov na Dyer (wameshatajwa) wanafafanua zaidi kuwa, mchakato huu hutokea kutegemea mahitaji ya kiwakati, kimazingira na kijamii pale ambapo tamaduni mbili tofauti au zaidi zinapokusana. Mathalani, mabadiliko ya sayansi, teknolojia na ugunduzi mbalimbali unaotokea katika jamii huweza kuchochea ukopaji. Jamii moja tuseme jamii ya lugha kopwaji inapofanya ugunduzi wa aina fulani jamii nyingine tuseme jamii ya lugha kopaji inapokabiliana na jamii iliyofanya ugunduzi wa vitu fulani hulazimika kukopa teknolojia hiyo na kuingiza katika lugha ya yake na kuitumia.

Ukopaji ni mchakato endelevu na unatokea katika lugha zote, hata kwa lugha ambazo hazina mnasaba, ilimradi tu makutano yatokee miongoni mwa lugha zinazohusika. Ndiyo maana lugha nyingi duniani zimepata kuathiriana kutokana na makutano yanayotokea katika lugha hizo. Tsvetkov na Dyer (wameshatajwa), wanatoa mfano kuwa lugha ya Kiswahili imekopa asilimia 40 ya msamiati wake kutoka katika lugha isiyo na mnasaba nayo ya Kiarabu. Schoen (keshatajwa) 
anafafanua kuwa lugha isiporuhusu mabadiliko kwa njia mbalimbali ikiwamo kukopa misamiati, basi kuna uwezekano wa lugha hiyo kupotea au kufa kabisa.

Buhumaid (2015) anafafanua kuwa ingawa kiisimu ukopaji unaonesha kutokea katika vipengele mbalimbali kama Tsvetkov na Dyer (2016) wanavyobainisha, lakini ukopaji wa kimsamiati ndio unafanyika zaidi kwa sababu yanafungamana kirahisi zaidi na mfumo wa lugha kopaji tofauti na ilivyo katika vipengele vingine vya lugha. Vilevile neno linaweza kutokea na kupotea haraka likiwa na madhara kiasi tu ya kisarufi. Maneno yenye manufaa katika lugha hupata mashiko zaidi, hivyo huwa ni vigumu kupotea. Mchakato unaohusishwa wa ukopaji wa kimsamiati ni mchakato wa kimofolojia, ambao ndio pia unachunguzwa katika makala haya. Maneno hujumuisha mofu huru na mofu tegemezi, ambazo ndizo zinaweza kuashiria hali ya ujinsi wa msamiati husika. Mofu huru huweza kuhamishwa bila ukomo kutoka lugha kopeshi kwenda katika kopaji, kwa kuwa hayo ni maneno kamili ya lugha yasiyo na kipingamizi cha kuingizwa katika lugha kopaji. Kwa upande mwingine, viambishi vya mofu tegemezi huhawilishwa kwenda lugha kopaji kwa kufuata ruwaza ya muundo au maandishi ya lugha hiyo (Filipović, 1990, kama alivyonukuliwa na Schoen, 2012). Hata hivyo, utaratibu wa ukopaji unatofautiana kutoka lugha moja hadi nyingine

\section{Nomino za Mkopo katika lugha ya Kiswahili}

Nomino mkopo inarejelea mchakato wa wazungumzaji kukopa nomino kutoka kwenye lugha kopwaji na kuingiza katika lugha kopaji. Shembilu (2017) anabainisha kuwa mchakato wa ukopaji wa nomino katika Kiswahili unahusisha michakato mikuu mitatu: udondoshaji, uchopekaji na usawazishaji wa maumbo. Kwa hiyo lugha ya Kiswahili inapokopa nomino kutoka lugha kopaji huingiza viambishi/sauti, kudondoshaji viambishi/sauti na kusawazisha sauti. Kama ilivyodokezwa hapo juu, kijinsi, lugha ya Kiswahili imewekwa katika lugha zisizokuwa na ujinsi katika nomino. Kwa mfano Morava na Kalarski (2013) wanabainisha hilo kuwa Kiswahili ni lugha isiyokuwa na jinsi katika msamiati wake. Wanafafanua zaidi kwamba, nomino za lugha ya Kiswahili zinajidhihirisha zaidi kimofosintaksia, kisemantiki, kimofolojia na kipragmatiki, pasipo kuashiria jinsi.. Ingawa inadaiwa kwamba lugha ya Kiswahili haina ujishi, tukichunguza nomino mbalimbali za mkopo zinazotumika katika lugha ya Kiswahili, hasa nomino za pekee, tunabaini kuwa kukiondolea ujinsi Kiswahili ni mtazamo wa jumla mno ambao unahitaji kusailiwa.

\section{Methodolojia}

Awali ya yote, mchunguzi aliandaa orodha ya majina na kuwapatia watoataarifa ili kuthibitisha taarifa zilizokuwa zinatafutwa. Majina hayo yalibainishwa na mtafiti kutegemea viashiria vya ujinsi vinavyoashiriwa na majina husika. Miongoni mwa viashiria hivyo ilikuwa mwelekeo wa kufanana kimofolojia. Yaani kuelekea kufanana katika mzizi wa neno husika na kutofautishwa kwa viambishi viwili au sauti moja tu lakini maana ikiwa ni tofauti. Watoataarifa watano (05) wanaosoma shahada ya umahiri mwaka wa pili katika Chuo Kikuu cha Dar es Salaam walifanyiwa mahojiano na mchunguzi kuhusiana na nomino hzo. Watoataarifa hao walipatikana kinasibu. Sababu za kufikiria kuwatumia watoataarifa wanaosoma shahada ya umahiri katika Kiswahili ilitokana na ukweli kwamba, wanafunzi hawa wamesoma kozi ya mofolojia ambayo ndani yake inazungumzia masuala ya ukopaji na taipolojia ya lugha. Hivyo, kwa kuwatumia watoataarifa hao ilichukuliwa kwamba wana uelewa wa masuala yanayochunguzwa na hivyo wangeliwezesha kupatikana kwa taarifa anazozitafuta. Majina yaliyobainishwa ni kama yanavyoonekana katika jedwali Na. 1.3. 
Jedwali Na. 1.3:

Nomino za Mkopo katika Kiswahili

\begin{tabular}{|l|c|}
\hline \multicolumn{1}{|c|}{ Na. } & Jina \\
\hline 1. & Khamisi/Hamisi? \\
\hline 2. & Hamisa \\
\hline 3. & Christiani /Kristiani? \\
\hline 4. & Christina Kristina? \\
\hline 5. & Fransisco/Fransisca \\
\hline 6. & Benedeta \\
\hline 7. & Saida \\
\hline 8. & Saidi \\
\hline 9. & Paulina \\
\hline 10. & Pauline /Paulini? \\
\hline 11. & Patrice /Patrisi? \\
\hline 12. & \\
\hline
\end{tabular}

Chanzo: Mtafiti (2018)

Jedwali $\mathrm{Na}$. 1.3 linaonesha nomino ambazo ni majina ya baadhi ya Waswahili ${ }^{3}$. Katika dodoso letu, watoataarifa walitakiwa kwanza kusema asili ya nomino ikiwa zinatokana na lugha za Kibantu au la. Katika kufanikisha lengo hili, mchunguzi aliandaa swali lililouliza; je, nomino zifuatazo zinatokana na lugha za Kibantu au za Kigeni? Swali la pili liliuliza hivi, kati ya nomino hizo ulizopewa, ni nomino gani zinaashiria jinsi ya kiume na zipi zinaashiria jinsi ya kike? Wekala alama ya vema panaophusika.

\section{Matokeo ya Uchunguzi}

Majibu kutokana na swali lililotaka kujua iwapo nomino zilizotolewa zinatokana na lugha za Kibantu au za kigeni yanaonesha kwamba hayo ni majina ya kigeni. Watoa taarifa wote watano walionesha kwamba, nomino hizo hazitokani na lugha za Kibantu, bali zimetokana na lugha za kigeni. Walifafanua zaidi kuwa nomino hizo zimeingia katika lugha ya Kiswahili kutokana na athari za kidini, yaani ama dini ya Kikristo au au dini ya Kiislamu. Aidha, kuhusu majibu ya swali lililotaka kujua ni nomino zipi kati ya zile zilizotolewa zinaashiria jinsi ya kiume na zipi zinaashiria jinsi ya kike, watoataarifa walionesha kama jedwali Na. 1.4 linavyoonesha:

Jedwali Na. 1.4:

Nomino za Mkopo katika Kiswahili kuonesha Jinsi

\begin{tabular}{|l|l|l|l|}
\hline Na. & \multicolumn{1}{|c|}{ Jina } & \multicolumn{2}{|c|}{ Jinsi } \\
\cline { 3 - 4 } & & Ke & Me \\
\hline 1. & Khamisi/Hamisi? & & $\sqrt{ } \sqrt{ }$ \\
\hline 2. & Hamisa & $\sqrt{ }$ & \\
\hline 3. & Christiani/Kristiani? & $\sqrt{ }$ & \\
\hline 4. & Christina/Kristina? & & $\sqrt{ }$ \\
\hline 5. & Benadicto/Benedicti /Benedikto? & & $\sqrt{ } \sqrt{ }$ \\
\hline 6. & Fransisco & & $\sqrt{ }$ \\
\hline
\end{tabular}

${ }^{3}$ Kulingana na BAKITA (2016, uk 744) Ni mtu wa Pwani ya Afrika ya Mashariki pamoja na visiwa vyake kwa kuzaliwa au kuwa na asili ya eneo hilo, na aliyeathirika na makuzi ya kiutamaduni (ikiwemo lugha) wa watu wa pwani pamoja na amali zao; ambapo lugha yake kuu ya mawasiliano ni Kiswahilli. 


\begin{tabular}{|l|l|l|l|}
\hline 7. & Fransinska & $\sqrt{ }$ & \\
\hline 8. & Benedeta & $\sqrt{ }$ & \\
\hline 9. & Saida & $\sqrt{ }$ & \\
\hline 10. & Saidi & & $\sqrt{ } \sqrt{ }$ \\
\hline 11. & Paulina & $\sqrt{ }$ & \\
\hline 12. & Pauline/Paulini? & & $\sqrt{ } \sqrt{ }$ \\
\hline 13. & Patrisi & $\sqrt{ }$ & $\sqrt{ }$ \\
\hline 14. & Patricia/Patrisia? & \\
\hline
\end{tabular}

Chanzo: Utafiti (2018)

Katika jedwali, alama ya $/ \sqrt{ } /$ ina maana jinsi ya kike na alma $/ \sqrt{ } /$ maana yake jinsi ya kiume. Uchunguzi unaonesha kuwa, nomino ya 1 na 2 kiasili ni neno moja ila yanatofautishwa kwa kuongeza viambishi -i ambacho kinaashiria jinsi ya kiume na kiambishi -a-, ambacho kinaashiria jinsi ya kiume. Pia, nominina ya 3 na 4 kiasili ni neno moja ila yametofautishwa kwa kuongezewa viamshi -i na -a vinavyoashiria jinsi katika lugha kopeshi. Vilevile, nomino katika namba 5 na 6 kimsingi ni moja katika lugha kopeshi. Katika lugha kopaji zimetofautishwa kwa kuongezewa viambishi -o, -i na -a zinzoashiria jinsi fulani. Hali ni hivyo hivyo kwa nomino namba 7, 8, 11 na 12. Aidha, nomino zilizopo katika mfano wa 9 na 10 viambishi -a na -e ndivyo vinatofautisha jinsi ya kiume na kike. Kiambishi -a kinaonesha jinsi ya kike ilhali kiambishi -e kinaonesha jinsi ya kiume. Mifano ni mingi lakini, kwa malengo ya makala haya, mifano hiyo inatosha. Kutokana na mifano hii, kuhitimisha kuwa lugha ya Kiswahili ni lugha isojinsi ni kitu kinachopaswa kutazamwa upya kwa kuzingatia nomino za mkopo zilizoingizwa katika lugha hii. Mifano hiyo ya data hapo juu inashabihiana na maelezo yaliyowahi kutolewa na mtaalamu Reza na wenzake (2016) kuwa ujinsi wa nomino kisarufi huathiri muundo wa maneno yanayohusiana nayo. Katika Kihispaniola kwa mfano vivumishi na viwakilishi hubailika muundo wake kutegemea nomino inayorejelewa. Nomino za Kihispaniola zina jinsi mbile, 'me' na 'ke' kama mfano wa nomino gato na gata. Nomino gato inaashiria jinsi 'me' na nomino gata inaashiria jinsi 'ke'.

Kama data katika Jedwali Na.1. 4 inavyoonesha, mabadiliko ya kiambishi kimoja yanabadili jinsi ya nomino inayohusika. Hali hiyo ni sawa na mfano wa nomino gato na gata katika lugha ya Kihspaniola ambapo fonimu moja tu ndiyo inafanya jinsi ya neno hilo iashirie ama 'me' au 'ke'. Hivyo, ni maoni ya makala haya kuwa, lugha ya Kiswahili si asilimia mia moja ni lugha isojinsi. Kwa kuwa imekuwa na makutano ya muda mrefu na lugha zenye jinsi katika nomino zake, na hivyo kuifanya ikope misamiati mingi katika lugha hizo. Athari za nomino mkopo zinaifanya lugha ya Kiswahili isiwe ni lugha isojinsi asilimia zote. Yawezekana utafiti mkubwa zaidi ukifanyika utabaini misamiati mingi zaidi yenye jinsi na pengine hata katika nyanja zingine kama vile fonolojia, semantiki na muundo. Makala yanatoa wito kwa wataalamu wengine kufanya uchunguzi wa kina zaidi kuhusiana na taipolojia ya lugha ya Kiswahili kijinsi ili kuweza kusaili mtazamo wa Morava na Kalarski (2013) kuhusu lugha hii.

\section{Hitimisho}

Makala haya yalilenga kuchunguza nomino za mkopo zinazoingia kutoka lugha nyingine huchukua tabia gani kijinsi. Matokeo ya uchunguzi yameonesha kuwa nomino za pekee za mkopo zinazoingia katika lugha ya Kiswahili huashiria jinsi. Nomino hiyohiyo moja ikipachikwa kiambishi cha aina fulani inaashiria jinsi ke na nomino hiyo ikibadilishwa kiambishi kingine anahama kategoria na kuashiria jinsi me. Makala haya yalishughulikia nomino kumi na mbili tu na zote zimeonesha tabia hiyo hiyo. Makala haya yanahitimisha kuwa, lugha ya Kiswahili siyo lugha isojinsi kwa asilimia mia moja. Mwito unatolewa kwa watafiti kuchunguza zaidi na kwa kina tabia ya nomino za mkopo zinazoingia katika lugha ya Kiswahili huwa na sifa zipi kitaipolojia. 


\section{Marejeleo}

Allen, J. V. (1993). Swahili origins: Swahili culture and the Shungwaya phenomenon. London: Ohio University Press.

Amidu, A. A. (1995). Kiswahili: People, Language, Literature and Lingua Franca. Nordic Journal of African Studies 4(1): uk.104-123 (1995).

Bahumaid, S. (2015). Lexical borrowing: The case of English loanwords in Hadhrami Arabic. International Journal of Language and Linguistics. 2, Na. 6; United Arab Emirates: University of Sharjah.

BAKITA, (2016). Kamusi kuu ya Kiswahili. Nairobi: Longhorn.

Filipović, R. (1990). Anglicizmi u hrvatskom ili srpskom jeziku. Zagreb: Školska knjiga.

Hultgren, A. (2017). New perspectives on language and gender: linguistic prescription and compliance in call centres. Language in Society, 1-25.

Massamba, D. P. B. (2002). Historia ya Kiswahili: 50 BK hadi 1500 BK. Nairobi: Jomo Kenyata Foundation.

Massamba, D. P. B. (2007). Kiswahili origins and the Bantu divergence-convergence theory. Dar es Salaam: TUKI.

Reza, P., Fahime, S., Laila, S., \& Tahereh, H. (2016). Examining the gender identity of language teachers using a masculinity-femininity scale: a case from Iran. Issues in Educational Research, 26(1), 2016: Chuo Kikuu cha Ferdowsi Mashhdad.

Schoen, E. (2012). Lexical borrowing in American sign language. Katika Intermediate ASL II SLS 2020: Michael Meldrum.

Shembilu, M (2017). Michakato ya kisemantiki kwenye maneno ya mkopo katika Kiswahili: mifano kutoka Kiingereza na kiarabu. Tasnifu ya uzamivu (haijachapishwa), Chuo Kikuu cha Dar es Salaam.

Tsvetkov, Y., \& Dyer, C. (2016). Cross-lingual bridges with models of lexical borrowing. Journal of Artificial Intelligence Research, 55 (2016) 63-93. Carnegie Mellon University. 